\title{
Erythema elevatum et diutinum assoziiert mit pulmonalen Infiltraten und nekrotisierender Keratopathie - eine diagnostische Herausforderung!
}

\author{
Erythema Elevatum et Diutinum Associated with Pulmonary Infiltrates \\ and Necrotizing Keratopathia - A Diagnostic Challenge!
}

Autoren

Institut
C. S. L. Müller, A. Wagner, C. Pföhler, W. G. Tilgen

Universitätsklinikum des Saarlandes, Klinik für Dermatologie, Venerologie und Allergologie, Homburg/Saar

\section{Bibliografie}

DOI http://dx.doi.org/

10.1055/s-0028-1119562

Online-Publikation: 13.3.2009

Akt Dermatol 2009; 35:

502-505 @ Georg Thieme

Verlag KG Stuttgart · New York ISSN 0340-2541

\section{Korrespondenzadresse}

Dr. med. Cornelia S. L. Müller Universitätsklinikum

des Saarlandes

Klinik für Dermatologie, Allergologie und Venerologie Kirrberger Straße 66421 Homburg/Saar hacmue@uniklinikumsaarland.de

\section{Zusammenfassung \\ $\nabla$}

Das Erythema elevatum et diutinum (EED) ist eine Erkrankung aus dem Kreis der neutrophilen Dermatosen. Die histologischen Befunde variieren mit Zeichen der leukozytoklastischen Vaskulitis in Frühstadien und einer konzentrischen Fibrose mit Lipidablagerungen in späten Stadien der Erkrankung. Einzelfälle einer Assoziation mit nekrotisierenden Lungenherden und entzündlichen Veränderungen verschiedener Augenabschnitte sind bekannt. Die Koinzidenz eines EED mit einer

\section{Anamnese}

Wir stellen eine 77-jährige Patientin vor, welche seit vier Jahren an indolenten Knoten im Bereich der unteren Extremitäten leidet. Diese zeigten in den letzten Wochen vor Erstkonsultation eine deutliche Tendenz zur Ulzeration. Die Hautveränderungen seien ausgehend von den Fersen zunehmend auch an den Unterschenkeln aufgetreten, zuletzt einhergehend mit einem süßlich-fötiden Geruch. Es bestand keine subjektive Beschwerdesymptomatik. Der Allgemeinzustand war jederzeit gut, ohne Fieber, Schüttelfrost oder Gewichtsverlust. Es waren keine konsumierenden Erkrankungen bekannt. Eine atopische Diathese lag nicht vor. Sie besaß keine Haustiere, kein Aquarium und keinen Gartenteich. Auslandsaufenthalte oder Fernreisen verneinte die Patientin. Zwei Monate vor der Vorstellung in unserer Klinik war eine stationäre Behandlung in einer Augenklinik notwendig geworden, bei spontan ulzerierend einschmelzender und perforierender Keratopathie des linken Auges mit konsekutivem vollständigem Visusverlust unklarer Genese. Seitdem trägt die Patientin eine Augenepi- nekrotisierenden Keratopathie und gleichzeitig nekrotisierenden bilateralen pulmonalen Herden ist jedoch bislang nicht beschrieben. Anhand der vorliegenden Krankengeschichte wird der ungewöhnliche klinisch-diagnostische Verlauf eines EED assoziiert mit bislang nicht beschriebenen nekrotisierenden pulmonalen Herden und einer nekrotisierenden Keratopathie diskutiert. Zur Diagnosefindung war die interdisziplinäre Zusammenarbeit mehrerer Fachgruppen und eine kritische abwägende Gewichtung von Klinik und Dermatohistologie erforderlich.

these links. Eine unter dem Verdacht auf eine Autoimmunkeratopathie durchgeführte Steroidpulstherapie zeigte weder eine Besserung der ophthalmologischen Problematik noch der kutanen Befunde.

\section{Aufnahmebefund}

$\nabla$

Bei Erstvorstellung sah man im Bereich beider Fersen (rechts > links) scharf begrenzte livide gefärbte, teils makulöse, jedoch überwiegend papulo-noduläre Läsionen mit teils ulzeriertem Zentrum und gelblich-hämorrhagischen Krusten. Insbesondere auf Druck kam es zu einer starken Exsudation von gelblich-hämorrhagischer Flüssigkeit. Fokal bestanden punktförmige purpuriforme Läsionen. An beiden Unterschenkeln fanden sich multiple, scharf abgrenzbare disseminiert verteilte, erythematöse, rundliche, infiltrierte und derb palpable Läsionen mit zentraler Ulzeration und Krustenbildung ( $\bullet$ Abb. 1 u. 2). Die umgebende Haut war unauffällig. 


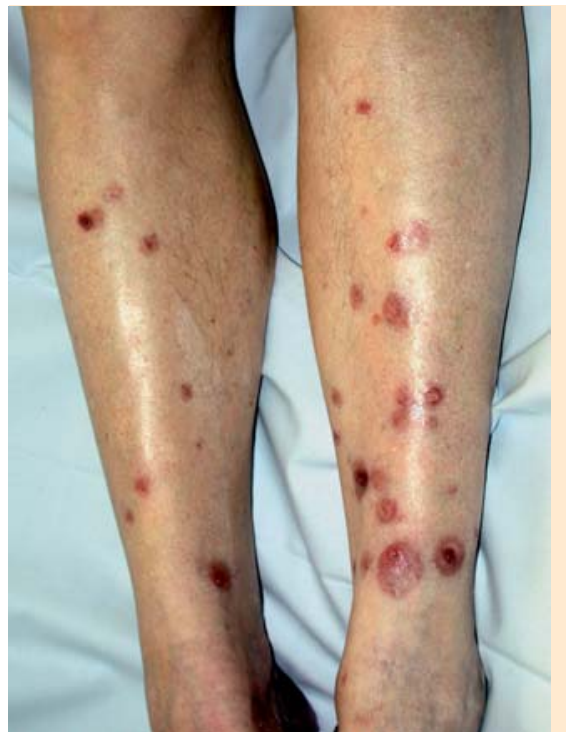

Abb. 1 77-jährige Patientin mit multiplen ulzerierten Läsionen, vor Therapiebeginn.

Abb. 2 77-jährige Patientin mit multiplen ulzerierten Läsionen, vor Therapiebeginn.

\section{Befunde diagnostischer Untersuchungen}

\section{Dermatohistologische Befunde}

Im Verlauf der Erkrankung wurden mehrfach Probebiopsien aus unterschiedlich frischen und älteren Läsionen zur dermatohistologischen Befundung entnommen. In einer floriden Läsion vom rechten Knie fand sich epidermal eine Spongiose und Akanthose mit fokaler Nekrose der Epidermis. Es bestand eine leukozytoklastische Vaskulitis der kleinen Gefäße der oberen und mittleren Dermis mit einem kräftig augeprägten keilförmigen Entzündungsinfiltrat aus neutrophilen Granulozyten mit Kernstaub, Makrophagen, Lymphozyten und zahlreichen Eosinophilen. Perivaskulär fand sich eine fibrinoide Degeneration. Granulome, Flammenfiguren oder Erreger waren nicht nachweisbar (๑ Abb. 3).

In einer älteren, klinisch fast abgeheilten Läsion vom Unterschenkel sah man mikroskopisch eine Kombination von Regeneratepithel und dermaler konzentrischer Fibrose ( $\bullet$ Abb. 4). Eine fibrinoide Degeneration war nicht mehr darstellbar, es fanden sich keine extrazellulären Cholesterol-Ablagerungen im fibrotischen Gewebe. Auch in der älteren Läsion waren in der PAS-Färbung keine Pilzelemente nachweisbar, ebenso ergab die ZiehlNeelsen-Färbung wiederholt keinen Nachweis säurefester Stäbchen. In der direkten Immunfluoreszenz konnten keine Immunglobulin-, Komplement- oder Fibrinablagerungen nachgewiesen werden.

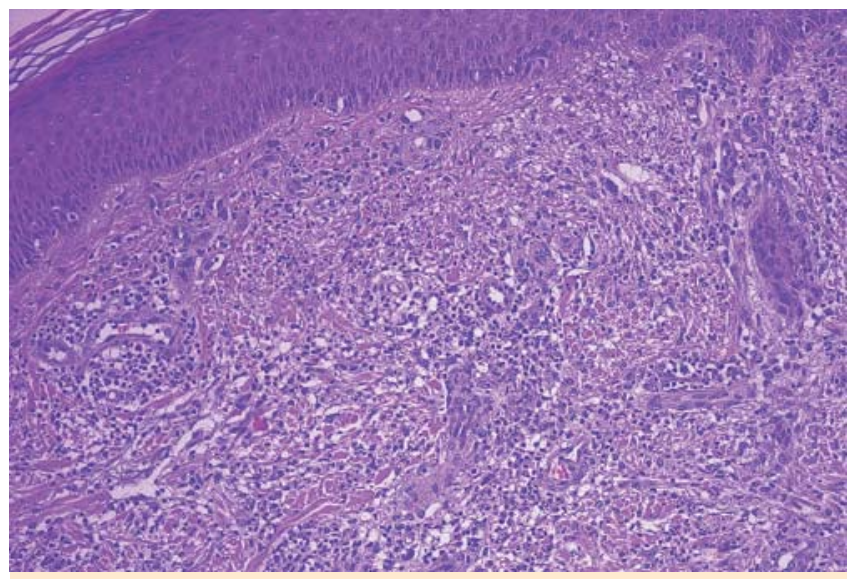

Abb. 3 (HE-Färbung, 20×). Leukozytoklastische Vaskulitis der kleinen Gefäße von oberer und mittlerer Dermis, dichtes und tiefreichendes Entzündungsinfiltrat aus neutrophilen Granulozyten mit Kernstaub, Makrophagen, Lymphozyten und zahllosen Eosinophilen.

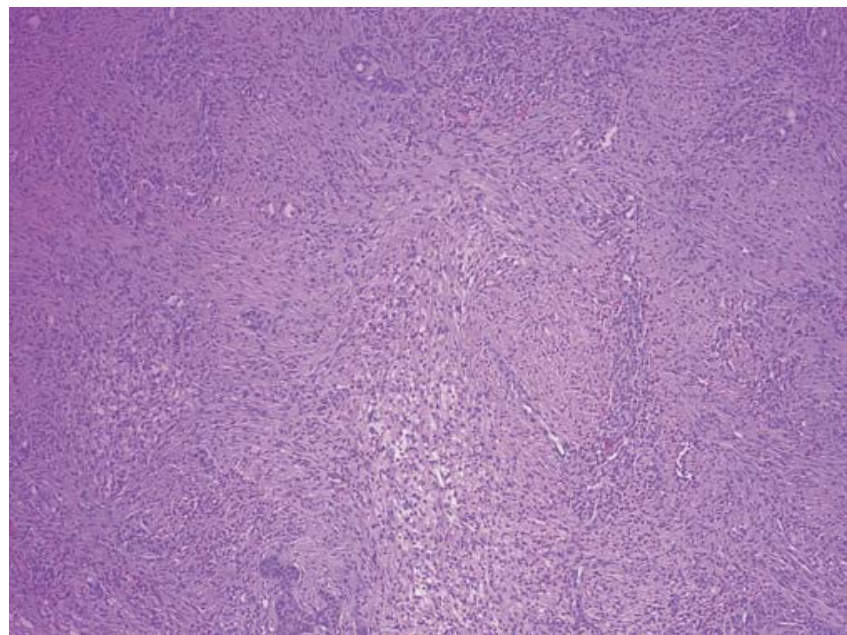

Abb. 4 (HE-Färbung, 10×). Spätes Stadium der Erkrankung mit konzentrischer Fibrose.

\section{Ergänzende Untersuchungen \\ $\nabla$}

Im Rahmen einer Durchuntersuchung wurden pulmonal multiple nekrotisierende Herde diagnostiziert, welche sich computertomografisch als abszedierende Einschmelzungen im Bereich beider Lungenunter- und -oberlappen darstellten. Radiologisch bestand kein Hinweis auf eine Neoplasie, spezifische Residuen, eine Pilzinfektion oder eine Wegener'sche Granulomatose.

Eine bronchoalveoläre Lavage, eine Bronchialaspirat-Zytologie und eine bronchiale Gewebsbiopsie erbrachten außer dem kulturellen Nachweis von Candida glabrata keine pathologischen Befunde. Histologisch, kulturell und molekularbiologisch bestand kein sicherer Anhalt für eine Mykobakterieninfektion. Die Gewebekultur und PCR aus bioptisch gesichertem Nativmaterial der Haut und paraffiniertem Gewebe ergab keinen pathologischen Befund. Aus mykologischen und bakteriellen Abstrichpräparaten der betroffenen Haut wurden Candida albicans und Candida glabrata sowie koagulase-negative Staphylokokken und Staphylokokkus aureus isoliert. Eine systemische Candidose wurde ausgeschlossen. Serologische Untersuchungen bzgl. Amöbiasis, Echinokokkose, Lues, Yersiniose, HIV und Hepatitis 
ergaben negative Befunde. Das übrige Routinelabor einschließlich Antistreptolysintiter war wiederholt unauffällig.

\section{Therapie und Verlauf}

Die Patientin bot bei stationärer Aufnahme einen Symptomenkomplex bestehend aus ulzerierenden Hautveränderungen an den Fersen und Unterschenkeln, einschmelzenden Lungenherden und einem Z.n. spontan ulzerierend einschmelzender und perforierender Keratopathie des linken Auges mit vollständigem Visusverlust.

\section{Differenzialdiagnostische Überlegungen}

Differenzialdiagnostisch wurden ein Pyoderma gangraenosum, ein tumorähnliches eosinophiles Granulom, eine kutane Candidose und eine atypische Mykobakteriose diskutiert:

Klinisch vereinbar mit den Hautbefunden war der seltene Fall eines multilokulären Pyoderma gangränosum mit pulmonaler Beteiligung [1,2]. Eine ulzerierende Keratitis in Assoziation mit einem Pyoderma gangraenosum ist in der Literatur bereits beschrieben [3].

Aufgrund der in der Hautbiopsie, im Lungenbiopsat sowie in der histopathologischen Befundung des enukleierten Auges nachgewiesenen Eosinophilie kam als weitere Differenzialdiagnose das sog. tumorähnliche eosinophile Granulom infrage. Es handelt sich um solitäre rot-braune Läsionen, zum Teil paraneoplastischer Genese, mit dem histologischen Befund einer tiefreichenden eosinophilendominierten Entzündungsreaktion ohne Nachweis von Flammenfiguren [4]. Jedoch wurden bei dieser sehr seltenen und bislang nur in 2 Fällen in der Weltliteratur beschriebenenen Entität bislang keine extrakutanen Manifestationen beobachtet [4]. Die nachgewiesene Infektion der kutanen Herde mit Candida albicans und spec. wurde als mykotische Superinfektion interpretiert. Der Nachweis von Candida glabrata im Bronchialaspirat sowie in der bronchoalveolären Lavage wurde durch die behandelnden internistischen Fachkollegen als nicht therapierelevant eingeordnet.

Das Vorliegen einer atypischen Mykobakteriose (aM) mit pulmonaler Beteiligung war in mehrerer Hinsicht zu diskutieren: Klinisch bestand der Aspekt einer sporotrichoiden Verteilung der Hautläsionen. Dermatohistologisch kann, auch bei fehlender granulomatöser Entzündung sowie negativem Erregernachweis in der Ziehl-Neelsen-Färbung, eine aM nicht sicher ausgeschlossen werden, da hierbei auch unspezifische histologische Befunde vorliegen können. Radiologisch konnte ebenso eine aM der Lunge nicht mit letzter Sicherheit ausgeschlossen werden. Mikrobiologisch konnten aus Gewebsmaterial DNA-Sequenzen amplifiziert werden, welche Sequenzhomologien zu bekannten atypischen Mykobakterien besaßen, sich jedoch nicht eindeutig einem bestimmten Erreger zuordnen ließen.

\section{Abschließende Beurteilung \\ $\nabla$}

Die o.g. feingeweblichen Befunde von Hautbiopsien sprachen für ein Erythema elevatum et diutinum. Eine im Vorfeld stattgehabte Steroidpulstherapie blieb ohne wegweisende Befundbesserung, sodass wir auf die Wiederholung einer Steroidtherapie verzichteten.

Zunächst erfolgte bei nachgewiesener mykotischer Infektion mit Candida albicans et spec. eine Therapie mit Fluconazol in einer

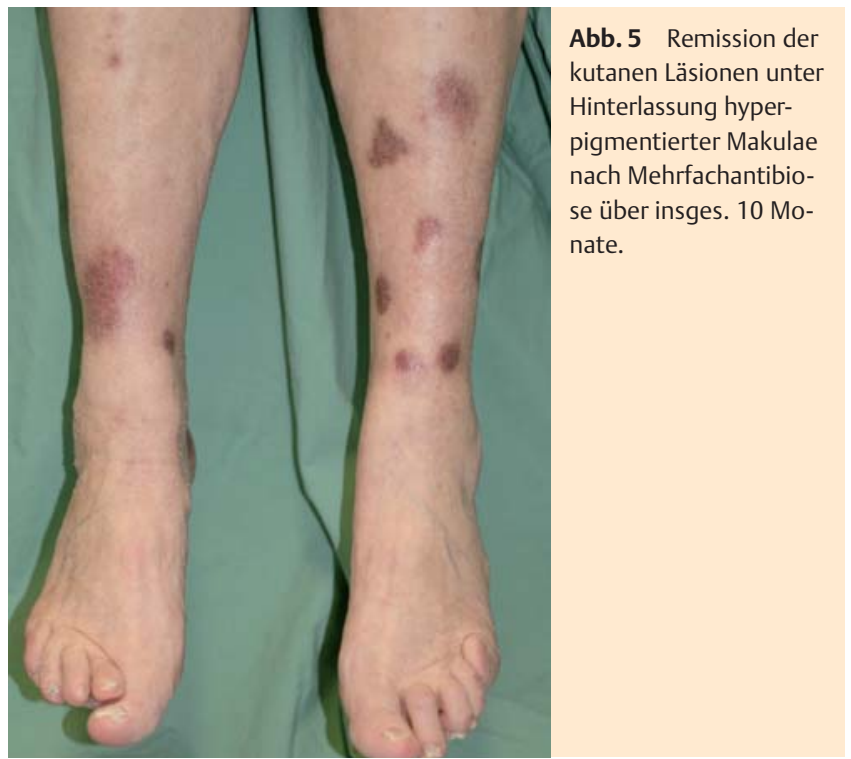

Dosierung von 200 mg über die Dauer von 3 Wochen bis zu dreimalig negativen Abstrichbefunden. Hierunter trat eine leichte Befundbesserung ein.

Bei bestehender klinischer und mikrobiologischer Differenzialdiagnose einer atypischen Mykobakteriose wurde im Anschluss hieran in Absprache mit den Kollegen der medizinischen Mikrobiologie und Hygiene eine antibiotische Kombinationstherapie mit Chlarithromycin, Mycobutin und Protionamid über insgesamt 10 Monate durchgeführt; initial wurde zusätzlich über 4 Wochen Imipenem gegeben.

Dies führte zu einer vollständigen Remission der kutanen Läsionen sowie einer Teilremission der pulmonalen Befunde bei letzter dermatologischer und radiologischer Verlaufsvorstellung ( Abb.5), sodass die Einleitung einer (klassischen) Therapie des EED mit Dapson nicht mehr erforderlich war.

\section{Diskussion}

$\nabla$

Das Krankheitsbild wurde erstmals durch Jonathan Hutchinson im Jahre 1880 sowie durch Judson Bury 1889 beschrieben [5 - 7]. Henry Radcliffe-Crocker gab der Erkrankung im Jahre 1894 den Namen Erythema elevatum et diutinum (EED) [7,8]. Es handelt sich um eine Erkrankung aus dem Formenkreis der neutrophilen Dermatosen, die sich durch meist symmetrisch auftretende, asymptomatische, bräunlich-erythematöse, papulo-noduläre Läsionen in Gelenknähe der Extremitäten (Knie oder Ellenbogen, Fingergelenke) auszeichnet. Lokalisationen am Körperstamm oder retroaurikulär wurden seltener beschrieben $[9,10]$. Die Hautläsionen zeigen eine stadienabhängige Ausprägung: Sehr frühe Läsionen sind von hellroter Farbe, spätere Läsionen lividrot bis rotbraun. Selten treten, wie in unserem Fall, Effloreszenzen in Form vesikulöser Läsionen, hämorrhagischer Knoten sowie Ulzerationen auf $[9,11]$. Abheilende Läsionen zeichnen sich durch einen gelblich-braunen Farbton aus, welcher klinisch an Xanthome erinnert [9]. In einigen Fällen wurde ein leichter Pruritus beschrieben. Pathogenetisch wird das EED aktuell zusammen mit dem Sweet-Syndrom, dem Pyoderma gangraenosum und der subkornealen pustulösen Dermatose zu den sog. neutrophilen Dermatosen gezählt. Eine kausale Beziehung zu vaskulären Immunkomplex-Ablagerungen wird diskutiert. In klassi- 
scher Weise werden sowohl Streptokokken-Infekte als auch rheumatologische Erkrankungen als Ursache für die Ausbildung von Immunkomplexen genannt [9]. In den vergangenen Jahren wurden jedoch zudem eine Vielzahl assoziierter bzw. zugrunde liegender Erkrankungen beschrieben, insbesondere Erkrankungen des hämatopoetischen Systems, die seropositive rheumatoide Arthritis, Morbus Crohn, Colitis ulzerosa, Pyoderma gangraenosum, Diabetes mellitus Typ 1 und die gluten-sensitive Enteropathie. Assoziierte infektiöse Erkrankungen sind in erster Linie bakterielle, virale, tuberkulöse Infektionen sowie die Lues $[9,12]$. Auch sind Fälle assoziierter Virushepatitiden sowie HIV beschrieben [9]. Die Hauterkrankung zeigt einen chronischen Verlauf über viele Jahre. Klinische Differenzialdiagnosen umfassen u. a. das Granuloma anulare, das Sweet-Syndrom, Non-Langerhanszell-Histiozytosen, das extrafaziale Granuloma eosinophilicum, Xanthome sowie die Sarkoidose [11].

Histologisch ist der Grundsatz „life of lesions“ zu beachten, mit typischen histomorphologischen Befunden in frühen Läsionen sowie in älteren Läsionen. Frühe Läsionen zeigen das typische Bild der „small-vessel-disease“ mit perivaskulären Infiltrationen durch neutrophile Granulozyten mit Leukozytoklasie, Makrophagen, Lymphozyten sowie eosinophilen Granulozyten, dermale Fibrinablagerungen und endotheliale Schwellung. Gefäßokklusionen können vorkommen. In seltenen Fällen können auch Veränderungen am bedeckenden Epithel in Form von Spongiose, Akanthose und Ulzerationen auftreten [12]. Reife, bzw. ältere Läsionen zeigen eine konzentrische dermale Fibrose mit einer persistierenden, wenngleich schütteren Entzündungsreaktion $[12,13]$. In lange bestehenden Läsionen kommt es zu extrazellulären Cholesterol-Ablagerungen im fibrotischen Gewebe [9]. Als histologische Variante dieser Cholesterolablagerungen wurde 1958 die extrazelluläre Cholesterinose Kerl-Urbach beschrieben, welche bereits zum damaligen Zeitpunkt als Variante des EED diskutiert wurde [14].

Histopathologische Differenzialdiagnosen umfassen das Granuloma eosinophilicum, das Sweet-Syndrom, andere Formen der leukozytoklastischen Vaskulitis und die neutrophile rheumatoide Dermatose. In älteren Läsionen müssen Dermatofibrome und Kaposi-Sarkome abgegrenzt werden $[9,11,13]$.

Es liegen mehrere Berichte über die Assoziation eines EED mit Augenerkrankungen vor: die marginale Hornhaut-Degeneration Fuchs-Terrien [16], periphere ulzerative Keratitiden [17,18], noduläre Scleritis und Panuveitis sowie autoimmune Keratolyse [19]. In der Literatur wird unseres Wissens bislang nur über einen einzigen Fall einer Assoziation eines EED mit pulmonalen Infiltraten berichtet [15]. In dieser Arbeit wurde das EED den neutrophilen Dermatosen zugerechnet, von denen einige eine Lungenbeteiligung aufweisen [2]. Während somit Einzelfälle einer Assoziation mit nekrotisierenden Lungenherden und entzündlichen Veränderungen verschiedener Augenabschnitte beschrieben sind, gibt es bislang keine Berichte über eine Koinzidenz eines ausgedehnten EED mit einer nekrotisierenden Keratopathie und nekrotisierenden bilateralen pulmonalen Herden.

\section{Erythema Elevatum et Diutinum Associated with Pulmonary Infiltrates and Necrotizing Keratopathia - A Diagnostic Challenge!}

Erythema elevatum et diutinum (EED) is subsumed in the group of neutrophilic dermatoses. Histological features display a leucocytoclastic vasculitis in early stages and concentric fibrosis in late stages of the disease. Associations to necrotizing pulmonary infiltrates or oculopathies are known. Co-incidence of an EED with necrotizing keratopathia and necrotizing bilateral pulmonary lesions has not been described yet.

\section{Literatur}

1 Schuppli R, Birkhauser $H$. A case of pyoderma gangraenosum with pulmonary changes. Dermatologica 1957; 115: 648-655

2 Vignon-Pennamen MD et al. Pyoderma gangrenosum with pulmonary involvement. Arch Dermatol 1989; 125: 1239-1242

3 Wilson DM, John GR, Callen JP. Peripheral ulcerative keratitis - an extracutaneous neutrophilic disorder: report of a patient with rheumatoid arthritis, pustular vasculitis, pyoderma gangrenosum, and Sweet's syndrome with an excellent response to cyclosporine therapy. J Am Acad Dermatol 1999; 40: 331 - 334

4 Gerbig AW, Zala L, Hunziker T. Tumorlike eosinophilic granuloma of the skin. Am J Dermatopathol 2000; 22: 75 - 78

5 Bury JS. A case of erythema with remarkable nodular thickening and induration of the skin associated with intermittent albuminuria. Illus Med News 1889; 3: 145

6 Hutchinson J. On two remarkable cases of symmetrical purple congestion of the skin in patches, with induration. British Journal of Dermatology $1880 ; 1: 10$

7 Iglesias-Gamarra A, Restrepo JF, Matteson EL. Small-vessel vasculitis. Curr Rheumatol Rep 2007; 9: 304-311

8 Radcliffe-Crocker HWC. Erithema elevatum diutinum. British Journal of Dermatology 1894; 6: 33-38

9 Gibson LE, el-Azhary RA. Erythema elevatum diutinum. Clin Dermatol 2000; 18: 295-199

10 Ly H, Black MM. Atypical presentation of erythema elevatum diutinum. Australas J Dermatol 2005; 46: 44-46

11 Gerbig AW, Zala L, Hunziker T. Erythema elevatum diutinum. A rare dermatosis with a broad spectrum of associated illnesses. Hautarzt 1997; 48: $113-117$

12 Wahl CE, Bouldin MB, Gibson LE. Erythema elevatum diutinum: clinical, histopathologic, and immunohistochemical characteristics of six patients. Am J Dermatopathol 2005; 27: $397-400$

13 High WA et al. Late-stage nodular erythema elevatum diutinum. J Am Acad Dermatol 2003; 49: 764-767

14 Herzberg JJ. Extracellular cholesterinosis of Kerl-Urbach, a variant of erythema elevatum diutinum. Arch Klin Exp Dermatol 1958; 205: 477-496

15 Creus L et al. Erythema elevatum diutinum associated with pulmonary infiltrates. Br J Dermatol 1997; 137: 652 - 653

16 Shimazaki J et al. Terrien's marginal degeneration associated with erythema elevatum diutinum. Cornea 1998; 17: 342 - 344

17 Takiwaki $H$ et al. Peripheral ulcerative keratitis associated with erythema elevatum diutinum and a positive rheumatoid factor: a report of three cases. Br J Dermatol 1998; 138: 893-897

18 Aldave AJ et al. Peripheral keratitis associated with erythema elevatum diutinum. Am J Ophthalmol 2003; 135: 389-390

19 Casanova FH et al. Autoimmune keratolysis in a patient with leukocytoclastic vasculitis: unusual erythema elevatum diutinum with granulomatous pattern. Cornea 2001; 20: 329-332 